\title{
Resonant production of fermions in an axial background
}

\author{
Antonio L. Maroto \\ Astronomy Centre, University of Sussex, Falmer, Brighton BN1 9QJ, United Kingdom \\ Anupam Mazumdar \\ Astronomy Centre, University of Sussex, Falmer, Brighton BN1 9QJ, United Kingdom \\ and Astrophysics Group, Imperial College, Blackett Laboratory, Prince Consort Road, London, SW7 2BZ, United Kingdom
}

(Received 10 November 1998; published 24 March 1999)

\begin{abstract}
We consider the resonant production of fermions from an oscillating axial background. The classical evolution of the axial field is given by that of a massive pseudovector field, as suggested by the renormalizability of the theory. We look upon both the massive and the massless fermion production from a perturbative point of view. We obtain the corresponding spectrum and angular distributions for the different spins or helicities in the particular case of a spatial-like axial field. We also extend our study to the nonperturbative regime in the massless case and compare the results with the perturbative ones. [S0556-2821(99)04308-8]
\end{abstract}

PACS number(s): $98.80 . \mathrm{Cq}$

\section{INTRODUCTION}

The parametric resonance phenomenon plays a fundamental role in the modern theories of reheating after inflation [1]. In such theories, after a period of inflation, driven generally by the potential energy of the slowly rolling inflaton field, the inflaton experiences a process of coherent oscillations around the minimum of the potential. Because of its coupling to the rest of the fields, those oscillations can give rise to the production of an exponentially large amount of bosons and subsequently to fermions through the decay of newly formed bosons, whose energy spectrum is characterized by resonance bands. Although it is an efficient mechanism to convert the inflaton energy to the intermediate bosons, it is still far from complete and we still require to invoke the old idea of reheating where the bosons decay to various fermions and that subsequently leads to the thermalization of the universe. The standard picture of preheating has not been tested for fields other than inflatons, and the effect of other fields that could be present in the early universe such as the dilaton, axial, or moduli predicted by string theory is still unexplored. It is a general belief that there would still be an amplification to one of the dominant fields depending upon the values of coupling constants but it has also been shown that for two scalar fields the system becomes chaotic [2].

The production of particles from classical sources has other important consequences apart from reheating. In fact, the same phenomenon is responsible for the amplification of vacuum fluctuations in the generation of gravitational waves [3]. It has also been proposed as a mechanism for the generation of primordial magnetic fields in the context of string cosmology [4]. In this latter model, a dynamical dilaton field plays the role of the external source. In addition, many other examples of particle production based on alternative gravity theories can be found in the literature, such as Brans-Dicke [5], higher dimensional models [6], etc.

In this paper we do not intend to give a holistic picture of preheating; rather we explore a different mechanism for the amplification of vacuum fluctuations: it is based on the presence of an oscillating background axial field. Axial (or antisymmetric) fields appear naturally in the bosonic sector of the low-energy string effective action together with graviton and dilaton fields $[7,8]$. In fact, recently some explicit solutions with nontrivial axion fields have been obtained in the context of homogeneous, isotropic, and anisotropic models in string cosmology $[9,10]$. These antisymmetric fields can be interpreted as torsion in pseudo-Riemannian geometry [8] and, as we will show, this fact provides a natural way of coupling them to other matter field. This is also the case of supergravity theories which contain a nonsymmetric part in the spin connection that is determined by the gravitino field [11].

The presence of general chiral fields can give rise to a different kind of production from the usual scalar fields. In particular, the breaking of parity invariance could lead to a different production of left and right fermions. In the present case, this will not happen because our model is parity invariant. In addition, as far as rotational invariance is broken by the axial field, we will obtain an anisotropic production of particles. In the usual picture of reheating it is expected that such initial anisotropy is rapidly erased by subsequent decays of the fermions produced in arbitrary directions. On the other hand, as far as the background field could have a nonvanishing angular momentum, we should also pay attention to the spin distribution of the produced particles. Again, in our case, we will consider a very simple homogeneous background with vanishing total angular momentum and we will not generate any net spin.

The paper is organized as follows. In Sec. II, we study the classical equations of motion for the axial field and its coupling to fermions. Section III is devoted to the perturbative calculation valid in the limit of small axial field for both massive and massless fermions. In Sec. IV we extend our calculation to the nonperturbative regime in the masless limit and compare our results with the previous ones. In Sec. V we include the main conclusions of the work.

\section{MINIMAL COUPLING AND THE AXIAL FIELD DYNAMICS}

The torsion field can be seen to be minimally coupled to fermions by means of the Einstein equivalence principle 
$[12,13]$. Thus, the Dirac Lagrangian for a single fermion field wih mass $m$ in the presence of curvature and torsion is given by

$$
\mathcal{L}=\bar{\psi}\left[i \gamma^{\mu}\left(\partial_{\mu}+\Omega_{\mu}+i g S_{\mu} \gamma_{5}\right)-m\right] \psi,
$$

where $S_{\rho}=\epsilon_{\mu \nu \lambda \rho} T^{\mu \nu \lambda}$ is the torsion pseudotrace, $\Omega_{\mu}$ the spin connection, and the coupling constant is fixed $g=1 / 8$ (we will work with a general $g$ in order to extend our results to other models in which $S_{\mu}$ is not interpreted as torsion). Despite the presence of the $\gamma_{5}$, the Lagrangian is parity invariant since $S_{\mu}$ is a pseudovector. As a consequence of this coupling, the axial field can act as a source of fermion creation. Notice that this coupling is dictated by the Einstein equivalence principle, unlike the usual inflaton couplings which are introduced ad hoc. The problem of fermion production has received attention only very recently in the works of Greene and Kofman [14] and Baacke et al. [15]. They have studied for the first time the problem of reheating of fermions from the coherent oscillations of the inflaton field. They showed how the limit on the occupation number imposed by Pauli blocking was saturated by the resonant production.

The first problem we have to deal with regarding the creation of particles is that of the classical dynamics of the torsion field. There are several models proposed in the literature for which torsion appears in the classical action only as a mass term; thus, for instance, the Einstein-Cartan theory considers the Einstein-Hilbert action but replaces the scalar curvature built out of the Levi-Cività connection by the scalar curvature built out of an arbitrary connection with torsion. In this theory torsion is a nonpropagating field that appears in the Lagrangian simply in a mass term of the form $M_{P}^{2} S^{2}$, with $M_{P}$ the Planck mass. This is also the case of the bosonic sector of the low-energy string effective action. However, if we consider the coupling of torsion to quantum fermionic fields in Eq. (1), the conditions of unitarity and renormalizability automatically require the existence of a kinetic term for the axial field, together with the previous mass term; i.e., they impose the axial field to behave like an Abelian massive axial vector $[16,17]$. In fact, the vacuum divergences generated by the above Lagrangian in Eq. (1) are given by

$$
S_{d i v}[S]=\frac{\Delta}{(4 \pi)^{2}} \int d^{4} x\left(-\frac{1}{192} S_{\mu \nu} S^{\mu \nu}+\frac{m^{2}}{16} S_{\mu} S^{\mu}\right),
$$

where $S_{\mu \nu}=\partial_{\mu} S_{\nu}-\partial_{\nu} S_{\mu}, \Delta=N_{\epsilon}+\log \left(\mu^{2} / m^{2}\right)$ with the poles parametrized as usual in dimensional regularization by $N_{\epsilon}$ $=2 / \epsilon+\log 4 \pi-\gamma$, and $\mu$ is the renormalization scale. Notice that there is no $S^{4}$ term although it has the same dimension. When instead of a single fermion we have more than one, the previous action is replaced by the sum of the actions corresponding to each single fermion. Therefore by an appropriate renormalization of mass and wave function we can take as the classical Lagrangian for torsion that of the Abelian massive gauge field. This is the minimal Lagrangian for torsion that ensures the renormalizability of the fermionic sector. This model is analogous to the inflaton model with a qua- dratic potential, but replacing the scalar field by a pseudovector. Notice that unlike the vector Abelian case, the presence of massive fermions is incompatible with a gauge invariance associated with the axial field. The corresponding equation of motion is nothing but the Proca equation, i.e.,

$$
\partial_{\mu} S^{\mu \nu}+m_{s}^{2} S^{\nu}=0,
$$

with $m_{s}$ the mass of torsion. It is now easy to find solutions for this equation. In particular, when torsion is spatial-like and only depends on time we have the following oscillatory solutions: $S^{i}(t)=\hat{S}^{i} \sin \left[m_{s}\left(t-t_{0}\right)\right]$, with $\hat{S}^{i}$ constants. As is well known, this kind of periodic functions can give rise to the parametric resonance pheonomenon.

As in the inflaton models, the production of particles induces the damping in the torsion oscillations. This can be described either by including a friction term in the equations of motion or by means of the vacuum polarization correction to the torsion mass. The vacuum polarization correction has been calculated in a previous paper [18] and from its imaginary part we get that

$$
\Gamma \equiv \frac{\operatorname{Im} \Pi\left(m_{s}\right)}{m_{s}} \propto \frac{g^{2} m^{2}}{m_{s}},
$$

where $m^{2}$ is the mass of the fermion squared, or in the case of several fermions present it is given by $m^{2}=\sum_{i} m_{i}^{2}$. Accordingly the classical evolution can be taken as

$$
S^{i}(t)=\hat{S}^{i} \sin \left[m_{s}\left(t-t_{0}\right)\right] e^{-\Gamma\left(t-t_{0}\right) / 2} .
$$

For the sake of simplicity we do not consider the expansion of the universe and accordingly all the calculations will be done in Minkowski space-time. We will also simplify further and take $S^{i}$ pointing in the $z$ direction. As usual in particle production calculations, we will impose our initial conditions on the background fields in such a way that it is possible to define asymptotic in and out vacuum states. With that purpose we will assume in next section that when $t \rightarrow-\infty$ the axial field is zero; i.e., we will take $S^{i}=0$ for $t<t_{0}$ and $S^{3}(t)$ given by Eq. (5) for $t \geqslant t_{0}$, as we will show that the result will not depend on the particular value of $t_{0}$ but only on the amplitiude of the oscillations $\hat{S}^{i}$ and decay rate $\Gamma$.

\section{PERTURBATIVE APPROACH}

\section{A. Massive fermions}

Let us consider the Dirac equation in the presence of the axial field:

$$
\left(i \gamma^{\mu} \partial_{\mu}-g S_{\mu} \gamma^{\mu} \gamma_{5}-m\right) \Psi=0 .
$$

It is to be noted that the form of the coupling does not allow us to disentangle the different spin modes, unlike in the case of Greene and Kofman. In addition, it cannot be reduced in an easy way to the known form of Mathieu equation or Lame equation, which have the known stability and the instability bands. Within instability bands the occupation number grows exponentially fast in the case of bosons. This keep us from finding the exact solution of the equation. However, it 
is possible to perform a perturbative analysis which provides part of the resonant behavior of the equation. There exists various approximation schemes to apply for the bosons as well as the fermions; we will follow that in [19]. Multiplying Eq. (6) by $\left(i \gamma^{\mu} \partial_{\mu}+g S_{\mu} \gamma^{\mu} \gamma_{5}+m\right)$, we get

$$
\begin{aligned}
& {\left[\partial_{\mu} \partial^{\mu}+i g \gamma^{\mu} \gamma^{\nu} \gamma_{5} \partial_{\mu} S_{\nu}+i g S_{\nu}\left\{\gamma^{\mu}, \gamma^{\nu}\right\} \gamma_{5} \partial_{\mu}-g^{2} S^{2}\right.} \\
& \left.\quad+2 g m \$ \gamma_{5}+m^{2}\right] \Psi=0 .
\end{aligned}
$$

Since there is no dependence on position in the Dirac operator, we can write the general solution $\Psi$ as

$$
\Psi(t, \vec{x})=\psi_{\vec{k}, s}(t) e^{i \vec{k} \cdot \vec{x}},
$$

where $s$ is a spin index. As we mentioned before we will take $S_{\mu} \equiv\left(0,0,0, S_{3}\right)$. Equation ( 7$)$ reduces to

$$
\begin{aligned}
\left(\partial_{0}^{2}+\vec{k}^{2}+i g \dot{S}_{3} \gamma^{0} \gamma^{3} \gamma_{5}-2 g k_{3} S_{3} \gamma_{5}+m^{2}+g^{2} S_{3}^{2}\right. \\
\left.+2 g m S_{3} \gamma^{3} \gamma_{5}\right) \psi_{k, s}(t)=0 .
\end{aligned}
$$

We introduce the plane-wave solutions for massive spinors with positive and negative frequency, and with a definite spin projection along the $z$ direction:

$$
\begin{aligned}
& U_{\vec{k}, s}(t, \vec{x})=\frac{1}{\sqrt{2 \omega}} u(\vec{k}, s) e^{i \vec{k} \cdot \vec{x}-i \omega t}, \\
& V_{\vec{k}, s}(t, \vec{x})=\frac{1}{\sqrt{2 \omega}} v(\vec{k}, s) e^{-i \vec{k} \cdot \vec{x}+i \omega t},
\end{aligned}
$$

where $s= \pm$ and $\omega^{2}=\vec{k}^{2}+m^{2} ; u(\vec{k}, s)$ and $v(\vec{k}, s)$ satisfy the following normalization conditions:

$$
\begin{aligned}
u^{\dagger}(\vec{k}, r) u(\vec{k}, s) & =2 \omega \delta_{r s}, \\
v^{\dagger}(\vec{k}, r) v(\vec{k}, s) & =2 \omega \delta_{r s}, \\
u^{\dagger}(-\vec{k}, r) v(\vec{k}, s) & =0, \\
v^{\dagger}(-\vec{k}, r) u(\vec{k}, s) & =0,
\end{aligned}
$$

where $u(\vec{k}, s)$ and $v(\vec{k}, s)$ are defined as

$$
\begin{gathered}
u(\vec{k}, s)=\sqrt{\omega+m}\left(\begin{array}{c}
\chi_{s} \\
\frac{\vec{\sigma} \cdot \vec{k}}{\omega+m} \chi_{s}
\end{array}\right), \\
v(\vec{k}, s)=\sqrt{\omega+m}\left(\begin{array}{c}
\frac{\vec{\sigma} \cdot \vec{k}}{\omega+m} \chi_{s} \\
\chi_{s}
\end{array}\right)
\end{gathered}
$$

and the Dirac spinors have the simple form

$$
\chi_{+}=\left(\begin{array}{l}
1 \\
0
\end{array}\right), \quad \chi_{-}=\left(\begin{array}{l}
0 \\
1
\end{array}\right) .
$$

We will work in this section in the Dirac representation for which the $\gamma$ matrices appear as

$$
\gamma^{0}=\left(\begin{array}{cc}
1 & 0 \\
0 & -1
\end{array}\right), \quad \gamma^{i}=\left(\begin{array}{cc}
0 & \sigma^{i} \\
-\sigma^{i} & 0
\end{array}\right), \quad \gamma_{5}=\left(\begin{array}{cc}
0 & -1 \\
-1 & 0
\end{array}\right) \text {. }
$$

Let us consider a given solution of the Dirac equation $\psi^{\text {in }}$ that initially, i.e., $t \rightarrow-\infty$, behaves like a plane wave with positive energy and spin $s$. In the remote future, where the interaction is switched off, such a solution will evolve into a linear combination of positive and negative frequences with a certain probability for spin flip, that is,

$$
\psi_{\vec{k} s}^{i n}(t, \vec{x}) \stackrel{t \rightarrow \infty}{\rightarrow} \sum_{s^{\prime}} \alpha_{\vec{k} s s^{\prime}} U_{\vec{k} s^{\prime}}+\beta_{\vec{k} s s^{\prime}} V_{-\vec{k} s^{\prime}} .
$$

The Bugolubov coefficients for fermions satisfy the following relation:

$$
\sum_{s^{\prime}}\left(\left|\alpha_{\vec{k} s s^{\prime}}\right|^{2}+\left|\beta_{\vec{k} s s^{\prime}}\right|^{2}\right)=1
$$

It is possible to transform the differential equation into an integral equation that allows a perturbative expansion

$$
\psi_{\vec{k} s}^{i n}(t, \vec{x})=U_{\vec{k} s}+\frac{1}{\omega} \int_{-\infty}^{t} M_{\vec{k}} \sin \left[\omega\left(t-t^{\prime}\right)\right] \psi_{\vec{k} s}^{i n}\left(t^{\prime}, \vec{x}\right) d t^{\prime}
$$

where the interaction term $M_{\vec{k}}$ is given by

$$
M_{\vec{k}}=\underbrace{g^{2} S_{3}^{2}}_{I}-\underbrace{2 g k_{3} S^{3} \gamma_{5}}_{I I}+\underbrace{2 g m \$ \gamma_{5}}_{I I I}+\underbrace{m^{2}}_{I V}+\underbrace{i g \dot{S}_{3} \gamma^{0} \gamma^{3} \gamma_{5}}_{V}
$$

From Eq. (19), we get for the Bugolubov coefficients to first order in perturbation theory the following expression:

$$
\beta_{\vec{k} s s^{\prime}}=V_{-\vec{k} s^{\prime}}^{\dagger} \psi_{\vec{k} s}^{i n}=-\frac{i}{4 \omega^{2}} \int_{-\infty}^{\infty} v_{-\vec{k} s^{\prime}}^{\dagger} M_{\vec{k}} u_{k s} e^{-i 2 \omega t^{\prime}} d t^{\prime},
$$

where we have substituted $\psi_{\vec{k} s}^{i n}$ on the right-hand side of Eq. (21) by the lowest-order solution $U_{\vec{k} s}$ and we have made use of the limit $t \rightarrow \infty$ of Eq. (21). The corresponding number of particles in a given spin state is given by the sum

$$
N_{\vec{k} s}=\sum_{s^{\prime}}\left|\beta_{\vec{k} s s^{\prime}}\right|^{2}
$$


Now we discuss the contribution of each term present in the potential. First we notice that the diagonal terms $I$ and $I V$ do not contribute to first order in perturbation theory. The $V$ term gives rise to

$$
v_{-\vec{k} s^{\prime}}^{\dagger} i g \dot{S}_{3} \gamma^{0} \gamma^{3} \gamma^{5} u_{\vec{k} s}=2 g \dot{S}_{3}\left(k_{2} \mp i k_{1}\right),
$$

where - sign applies for $s^{\prime}=-$ and $s=+$ and the + sign for opposite spin directions. For $s^{\prime}=s$ the term vanishes. The $I I$ term gives rise to

$$
-2 g v_{-\vec{k} s}^{\dagger} k_{3} S_{3} \gamma_{5} u_{\vec{k} s}=2 g k_{3} S_{3}\left(\omega+m-\frac{\omega^{2}-m^{2}}{\omega+m}\right) .
$$

Finally the $I I I$ term yields

$$
2 g m v_{-\overrightarrow{k s}}^{\dagger} S_{3} \gamma^{3} \gamma_{5} u_{\vec{k} s}=-4 g m S_{3} k_{3} .
$$

Notice that the contributions from $I I$ and III cancel each other and finally only the term $V$ contributes to the Bogolyubov coefficients. Performing the Fourier transform implicit in Eq. (23), and using the spherical coordinates in momentum space $(k, \theta, \phi)$, we can write the total number of particles produced with spin $s= \pm$ and momentum $\vec{k}$ as

$$
N_{\vec{k}+}=N_{\vec{k}-}=g^{2}\left|\hat{S}_{3}(\omega)\right|^{2} \frac{\vec{k}^{2}}{\omega^{2}} \sin ^{2} \theta
$$

with $\hat{S}^{3}(\omega)$ the Fourier transform of $S^{3}(t)$ and $\theta$ the angle between $\vec{k}$ and $\vec{S}$. As commented before, the number of produced fermions with spin up equals the number of fermions with spin down and as a consequence there is no net spin creation. The total number of fermions is just twice the above result.

We see that the production is not isotropic, with a maximum in the orthogonal directions to $\vec{S}(\theta=\pi / 2)$, and no forward-backward production $(\theta=0, \pi)$. In Fig. 1 we have plotted the spectrum in the lower curve and we observe the resonance close to $k=m_{s} / 2$ which corresponds to the decay of the axial field quanta into fermion-antifermion pairs.

\section{B. Massless fermions}

As we have seen, it is not possible to separate out the positive and negative spin states in the massive case, but in the massless case it is possible to do that by choosing a different representation for the Dirac matrices, the Weyl representation. Since the solutions of the Weyl equation in the absence of external sources can be written as eigenstates of the helicity operator, it is useful to consider those eigenstates for the positive and negative frequency solutions. We shall derive the total number of massless fermion production and we shall see that the summation of both chiralities gives rise to the total number of fermions and it is equal to the one obtained in the previous section taking $m \rightarrow 0$. The form of the plane-wave solutions with positive and negative frequency is that given in Eq. (11), but now the form of the spinors is given by

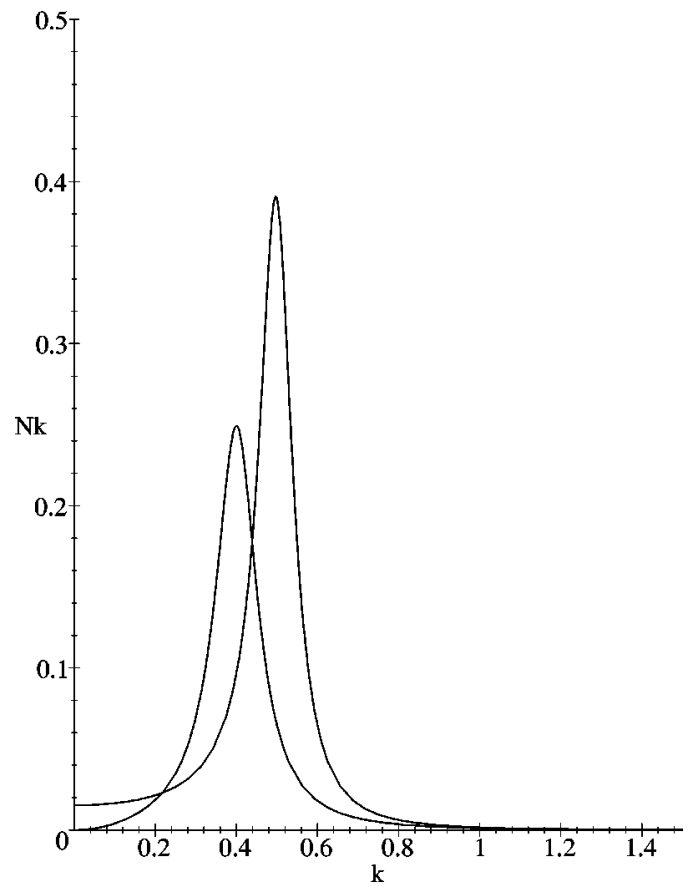

FIG. 1. Number of particles against $|\vec{k}|$ for $g \hat{S}=1 / 8 m_{s}$ and $\Gamma$ $=0.2 m_{s}$. For massive fermions $m=0.3 m_{s}$.

$$
\begin{aligned}
& u_{\vec{k}+}=\sqrt{2 \omega}\left(\begin{array}{c}
a_{+}(\vec{k}) \\
0
\end{array}\right), \quad u_{\vec{k}-}=\sqrt{2 \omega}\left(\begin{array}{c}
0 \\
a_{-}(\vec{k})
\end{array}\right), \\
& v_{\vec{k}+}=\sqrt{2 \omega}\left(\begin{array}{c}
b_{+}(\vec{k}) \\
0
\end{array}\right), \quad v_{\vec{k}-}=\sqrt{2 \omega}\left(\begin{array}{c}
0 \\
b_{-}(\vec{k})
\end{array}\right),
\end{aligned}
$$

where

$$
\begin{aligned}
& a_{+}(\vec{k})=-b_{+}(\vec{k})=\left(\begin{array}{c}
\cos \frac{\theta}{2} \\
\theta \\
\cos \frac{\theta}{2} e^{i \phi}
\end{array}\right), \\
& a_{-}(\vec{k})=-b_{-}(\vec{k})=\left(\begin{array}{c}
-\sin \frac{\theta}{2} e^{-i \phi} \\
\cos \frac{\theta}{2}
\end{array}\right)
\end{aligned}
$$

are eigenstates of the helicity operator with eigenvalues +1 and -1 , respectively. The normalization conditions are the same as the massive ones. In the Weyl representation the gamma matrices are

$$
\gamma^{0}=\left(\begin{array}{cc}
0 & -1 \\
-1 & 0
\end{array}\right), \quad \gamma^{i}=\left(\begin{array}{cc}
0 & \sigma^{i} \\
-\sigma^{i} & 0
\end{array}\right), \quad \gamma_{5}=\left(\begin{array}{cc}
1 & 0 \\
0 & -1
\end{array}\right) .
$$

In the present case, the initial positive frequency solution will evolve into a linear combination of positive and negative 
frequency modes, but since the perturbation is diagonal in chirality space, there is no mixing between positive and negative chirality modes, i.e.,

$$
\psi_{\vec{k} s}^{i n}(t, \vec{x}) \stackrel{t \rightarrow \infty}{\rightarrow} \alpha_{\vec{k} s} U_{\vec{k} s}+\beta_{\vec{k} s} V_{-\vec{k} s} .
$$

In the massless case, the contributions of the $I I I$ and $I V$ terms are not present and again the diagonal terms do not contribute. As a consequence only the $V$ term is relevant. Hence the total number of fermions with momentum $\vec{k}$ and helicity $s$ is given by the following expression:

$$
N_{\vec{k} s}=\left|\beta_{\vec{k}, s}\right|^{2}=g^{2} \sin ^{2} \theta\left|\hat{S}_{3}(\omega)\right|^{2} .
$$

The total number with both the spins would be just twice the above expression, which is the same as the massive case. In Fig. 1 we have represented the spectrum for massless fields in the upper curve. It is to be noted that the production of massive fermions is, as expected, suppressed by the mass term. We also observe the peak at $k=m_{s} / 2$.

\section{NONPERTURBATIVE RESULTS}

In the previous section we have obtained the spectrum and angular distribution of the produced particles up to first order in perturbation theory. Higher-order terms are expected to be suppressed and only will give rise to smaller peaks at higher energies. However, nonperturbative effects can have a more important effect on the results. As shown in the scalar case, the features of production essentially deviate from the pertubative result. Since in our case the equations cannot be reduced to any known form, we will solve them numerically. For the sake of simplicity we will only consider the massless case in which it is possible to disentangle the two spin states. We will also assume that the behavior of the background field is the following: for $t<0$ and $t>n T, S_{3}=\hat{S}$ a constant, with $n$ an integer and $T=2 \pi / m_{s}$ the period of the oscillations. For $0<t<n T, S_{3}=\hat{S} \cos \left(m_{s} t\right)$. It has been checked that the final spectra do not depend on $n$. In order to reduce Eq. (6) to a second order form we make the following ansatz on the solutions [14]:

$$
\psi=\left(i \not b+g \$ \gamma_{5}\right) f_{\vec{k} s}(t) e^{i \vec{k} \cdot \vec{x}} W_{s \pm},
$$

where for positive (negative) $+(-)$ energy modes with spin $s$ we take

$$
W_{s+}=\left(\begin{array}{c}
\chi_{s} \\
0
\end{array}\right), \quad W_{s-}=\left(\begin{array}{c}
0 \\
\chi_{s}
\end{array}\right)
$$

In this section we will work again in the Weyl representation. With this ansatz, the equations of motion can be written as a single equation for the function $f_{\vec{k} s}$ :

$$
\left(\partial_{0}^{2}+\vec{k}^{2}-i s g \dot{S}_{3}+2 g k_{3} S_{3}+g^{2} S_{3}^{2}\right) f_{\vec{k} s}(t)=0 .
$$

In addition the $\psi$ spinors will give rise asympotically $(t \rightarrow$ $\pm \infty$ ) to eigenstates of the the third component of the spin operator. In this sense, they have the appropriate form to describe the creation of fermions with definite spin along that direction. The normalized spectrum of the total number of particles created with spin $s$ is given by (see [20,21] for details)

$$
\begin{aligned}
N_{\vec{k} s} & =\frac{k_{1}^{2}+k_{2}^{2}}{\vec{k}^{2}+g^{2} \hat{S}^{2}+2 g \hat{S} k_{3}} \frac{1}{\sin ^{2}\left(d_{2}\right)}\left[\operatorname{Im} F_{\vec{k} s}(T)\right]^{2} \\
& =\frac{k^{2} \sin ^{2}(\theta)}{k^{2}+g^{2} \hat{S}^{2}-2 g \hat{S} k \cos \theta} \frac{\left[\operatorname{Im} F_{\vec{k} s}(T)\right]^{2}}{1-\left[\operatorname{Re} F_{\vec{k} s}(T)\right]^{2}},
\end{aligned}
$$

where $F_{\vec{k} s}$ is a solution of Eq. (37) with initial conditions $F_{\vec{k} s}(0)=1$ and $\dot{F}_{\vec{k} s}(0)=0$ and we have used the fact that $\cos d_{2}=\operatorname{Re} F_{\vec{k} s}(T)$. The numerical computations show that the number of fermions produced with spin up is the same as those with spin down; as expected there is no net spin creation in this particularly simple model. We see that the $\sin ^{2} \theta$ angular dependence that we obtained in the perturbative calculation is already present in the above result for $\hat{S} \gg k$, although it is expected to be modified by the factor involving $F_{\vec{k} s}(T)$. In Figs. 2 and 3 we have represented the spectra against $|\vec{k}|$ and $\theta$ for different values of the amplitudes. First we notice that for small amplitudes of the oscillations $\hat{S}$ there appears a peak at $k=0.5 m_{s}$ in agreement with the perturbative calculation. However, we find new peaks also for small amplitudes. For large values of the amplitude the departure from the perturbative results is apparent. We can also observe the appearance of the resonance bands in the spectra whose structure strongly depends on the angle $\theta$. In addition, the angular dependence is also highly sensitive to the energy $k$ of the fermions and the amplitude $\hat{S}$ of the oscillations. In particular we find that for large amplitude of the oscillations, strong peaks appear at some particular values of the angles, indicating that the production mainly takes place in those directions.

\section{CONCLUSIONS}

In this work we have studied the resonant production of fermions from a classical oscillating axial background. The dynamics of the classical background is determined by the renormalizabililty of the quantum theory of fermions coupled to axial fields and is given by the Proca equation. The oscillatory solutions to this equation are obtained and the corresponding phenomenon of resonant particle production is analyzed both from a perturbative and a nonperturbative point of view. In the perturbative case, we have considered both the massive and massless fermion cases for a simple background in which the axial field is oscillating in the $z$ direction, and we have obtained the spectra and angular distributions of the produced particles. In the nonperturbative case, we consider only the massless case and study the equation numerically. We have checked that the nonperturbative results deviate from the perturbative ones with the appearance of typical resonance bands, which in our case depend on both momenta and angle.

Concerning the possibility that this mechanism could 


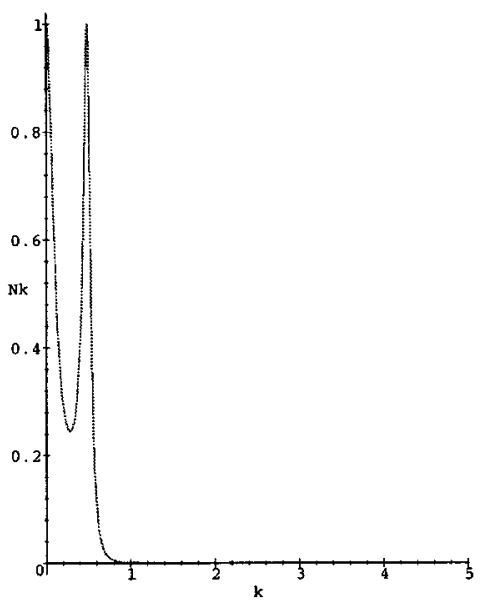

(a)

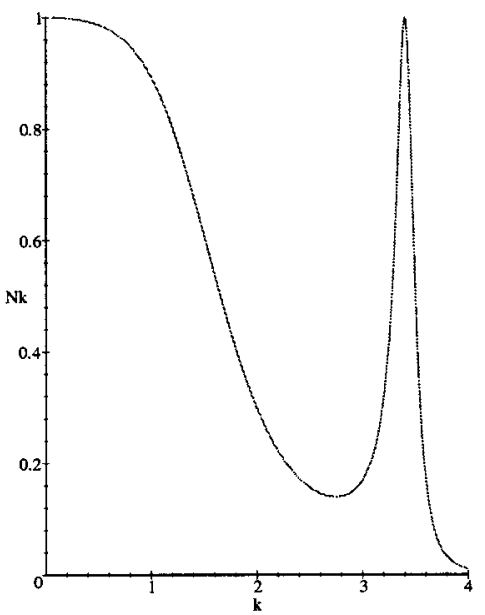

(c)

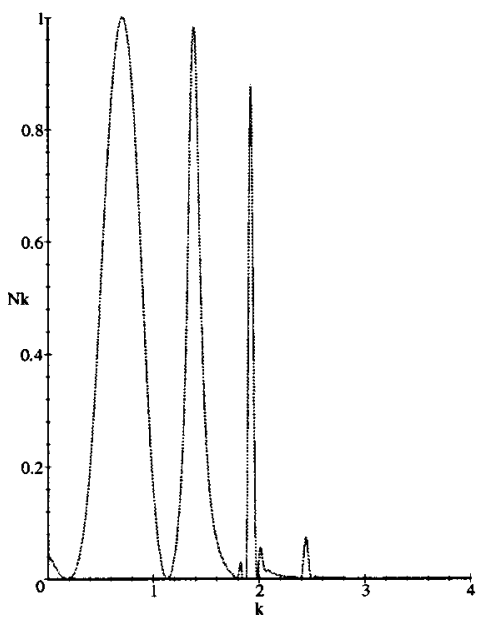

(e)

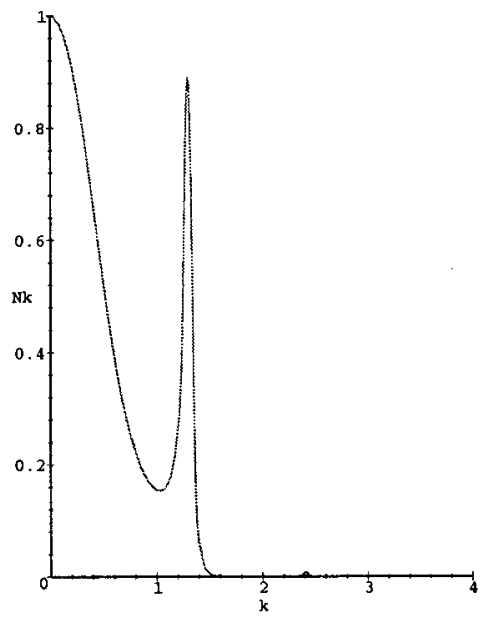

(b)

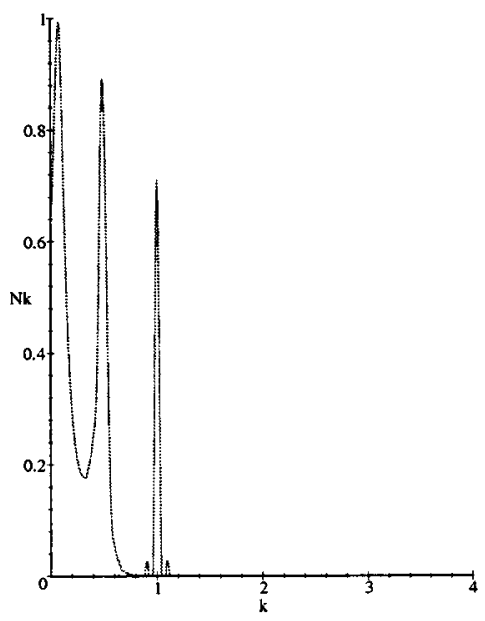

(d)

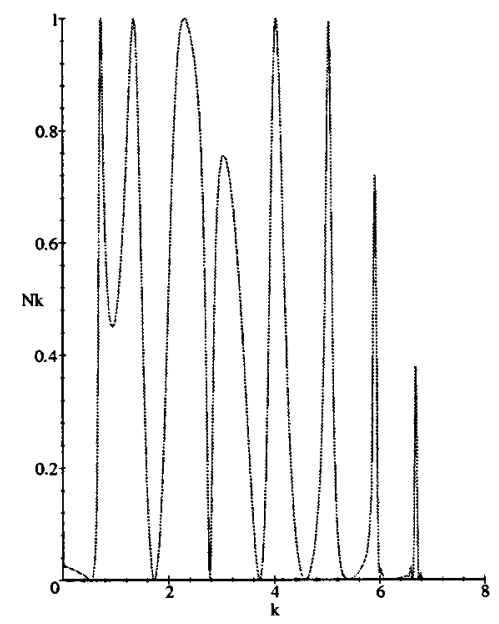

(f)

FIG. 2. Normalized number of particles against $|\vec{k}|$. (a), (b), and (c) correspond to $g \hat{S}=0.1 m_{s}, 1 m_{s}$, and $10 m_{s}$, respectively, with $\theta$ $=\pi / 2$ and (d), (e), and (f) to the same amplitudes but for $\theta=\pi / 4$. 


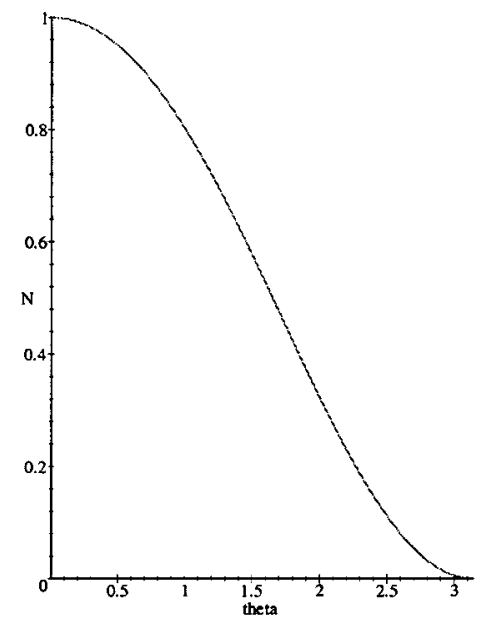

(a)

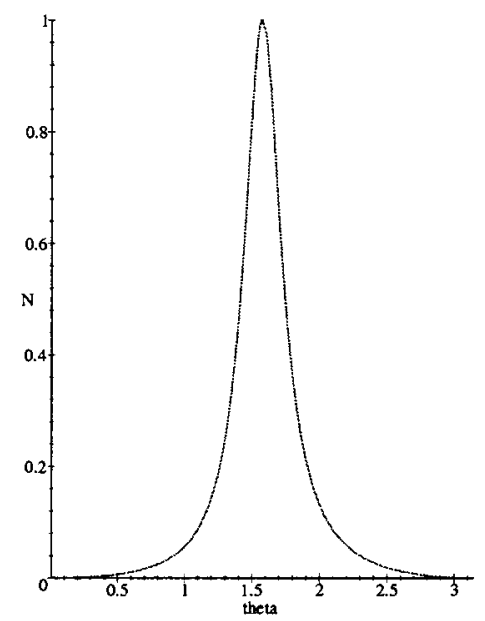

(c)

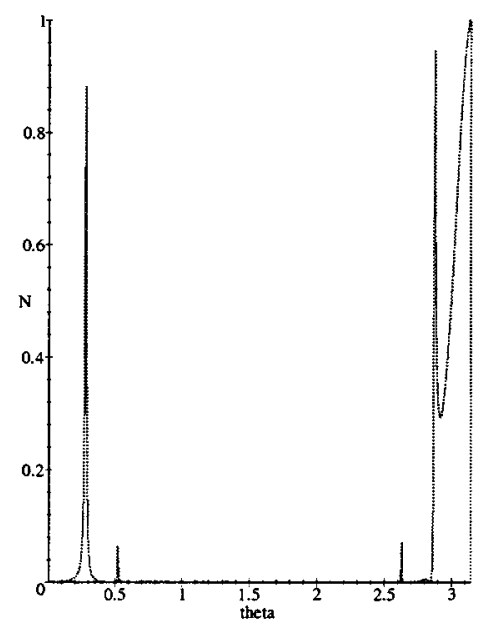

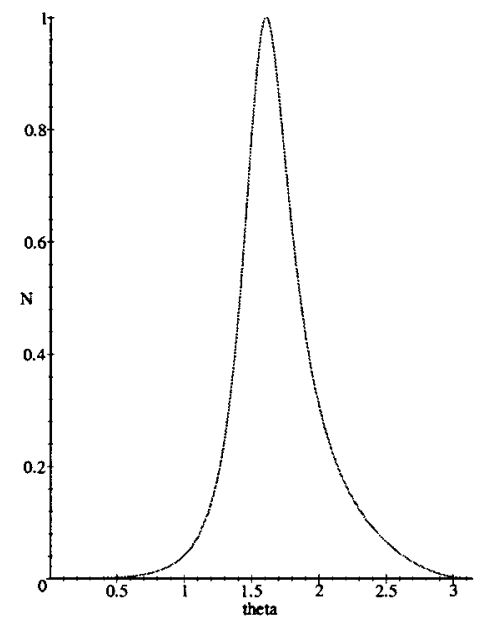

(b)

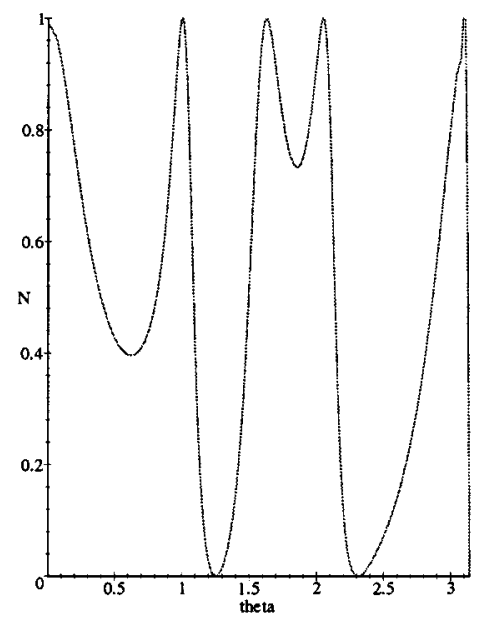

(d)

\section{(e)}

FIG. 3. Normalized number of particles against $\theta$. (a), (b), and (c) correspond to $g \hat{S}=0.1 m_{s}, 1 m_{s}$, and $10 m_{s}$, respectively, with $|\vec{k}|=0.1 m_{s}$ and (d), (e) to $g \hat{S}=10 m_{s}$ with $|\vec{k}|=1 m_{s}, 10 m_{s}$, respectively. 
have been relevant in the early universe, we should mention that the generation of an initial anisotropic axial field that later on could have evolved according to Eq. (3) has been explored in [22]. There it is shown that for axial fields interacting with photons, there is the possibility of spontaneous symmetry breaking along spatial directions for the axial field. As a consequence, that field would acquire some spatial-like vacuum expectation value. In addition, as we mentioned in the Introduction, axial fields are present in the context of string cosmology and therefore it would be very interesting to study if the resonant phenomenon studied in this work could affect the standard picture of reheating after inflation.

\section{ACKNOWLEDGMENTS}

A.L.M. acknowledges support from SEUID-Royal Society and (CICYT-AEN96-1634) (Spain). A.M. is supported by INLAKS and ORS. We thank Juan García Bellido, Luis Mendes, and Andrew Liddle for valuable discussions.
[1] L. Kofman, A.D. Linde, and A.A. Starobinsky, Phys. Rev. Lett. 73, 3195 (1994); L. Kofman, A.D. Linde, and A.A. Starobinsky, Phys. Rev. D 56, 3258 (1997).

[2] R. Easther and K. Maeda, gr-qc/9711035, 1997.

[3] L.P. Grishchuk, Sov. Phys. JETP 40, 409 (1975); Ann. (N.Y.) Acad. Sci. 302, 439 (1977).

[4] M. Gasperini, M. Giovannini, and G. Veneziano, Phys. Rev. Lett. 75, 3796 (1995).

[5] M. Gasperini and M. Giovannini, Phys. Rev. D 47, 1519 (1993).

[6] J. Garriga and E. Verdaguer, Phys. Rev. D 39, 1072 (1989).

[7] M.B. Green, J.H. Schwarz, and E. Witten, Superstring Theory (Cambridge University Press, Cambridge, England, 1987).

[8] R.R. Metsaev and A.A. Tseytlin, Nucl. Phys. B293, 92 (1987).

[9] E.J. Copeland, A. Lahiri, and D. Wands, Phys. Rev. D 51, 1569 (1995); 50, 4868 (1994).

[10] A.L. Maroto and I.L. Shapiro, Phys. Lett. B 414, 34 (1997).

[11] P. van Nieuwenhuizen, Phys. Rep. 68, 4 (1981).

[12] F.W. Hehl, P. Heyde, G.D. Kerlick, and J.M. Nester,
Rev. Mod. Phys. 48, 393 (1976).

[13] A. Dobado and A.L. Maroto, Phys. Rev. D 54, 5185 (1996).

[14] P.B. Greene and L. Kofman, hep-ph/9807339.

[15] J. Baacke, K. Heitmann, and C. Patzold, Phys. Rev. D 58, 125 013 (1998).

[16] A.S. Belyaev and I.L Shapiro, Phys. Lett. B 425, 246 (1998).

[17] A. Dobado and A.L. Maroto, hep-th/9712198.

[18] A.L. Maroto, Phys. Rev. D 59, 063501 (1999).

[19] N.D. Birrell and P.C.W. Davies, Quantum Fields in Curved Space (Cambridge University Press, Cambridge, England, 1982).

[20] V.M. Mostepanenko and V.M. Frolov, Sov. J. Nucl. Phys. 19, 451 (1974).

[21] A.A. Grib, S.G. Mamayev, and V.M. Mostepanenko, Vacuum Quantum Effects in Strong Fields (Friedmann Laboratory Publishing, St. Petersburg, 1994).

[22] A.A. Andrianov and R. Soldati, Phys. Lett. B 435, 449 (1998); A.A. Andrianov, R. Soldati, and L. Sorbo, Phys. Rev. D 59, 025002 (1999). 